HNO 2012 $\cdot 60: 770-770$

DOI 10.1007/s00106-012-2520-3

Online publiziert: 5. September 2012

(c) Springer-Verlag 2012

\title{
N. Stasche
}

HNO-Klinik, Schlafmedizinisches Zentrum,Westpfalz-Klinikum, Kaiserslautern

\section{Entfernung von Fremdkörpern}

den und steht häufig erst an zweiter Stelle beim Versagen einer Fremdkörperentfernung mit flexiblen Endoskopen. Wegen der speziellen Beatmungssituation ist die starre Trachebronchoskopie zur Entfernung aspirierter Fremdkörper nach wie vor ein sicheres Verfahren.

Komplikationen wie Hypopharynx- oder Ösophagusperforation oder eine sekundäre Aspiration zu vermeiden. Heute lassen sich mit den modernen flexiblen und klassischen starren endoskopischen Techniken nahezu alle Fremdkörper sicher aus dem oberen Gastrointestinaltrakt bergen. Dies ist insbesondere bei allen scharfkantigen und großen Fremdkörpern und bei ingestierten Batterien zwingend notwendig.

Aspirierte Fremdkörper stellen bei Lokalisation im Larynx oder in der Trachea eine Notfallsituation dar, bei der es auf ein schnelles Handeln ankommt. Andererseits können aspirierte Fremdkörper im Kindesalter nach Sistieren der anfänglich heftigen Hustenattacken leicht übersehen werden. Während nicht alle ingestierten Fremdkörper entfernt werden müssen, stellt der begründete Verdacht auf Fremdkörperaspiration eine Indikation zur bronchoskopischen Fremdkörperentfernung dar. Die Entscheidung zur endoskopischen Intervention stellt immer eine sehr individuelle Risikoabwägung zwischen Eingriffsrisiko und den potenziell schwerwiegenden Folgen des übersehenen Fremdkörpers dar. Dank moderner flexibler Endoskope mit dem immer besser werdenden Zusatzinstrumentarium, welches aus dem Fundus der interventionellen Endoskopie des Gastrointestinaltrakts entlehnt werden kann, ist die Dominanz der starren Fremdkörperendoskopie des Ösophagus nicht mehr vorhan-

\section{\) Aspirierte Fremdkörper können nach Sistieren der Hustenattacken leicht übersehen werden}

Die endoskopische Entfernung von Fremdkörpern im oberen Aerodigestivtrakt ist im HNO-Fachgebiet aufgrund seiner historischen Entwicklung und der Abbildung in der Weiterbildungsordnung nach wie vor fest verankert. Fremdkörperingestionen führen durch die damit verbundenen Schluckbeschwerden die Patienten häufiger primär zum niedergelassenen HNO-Arzt. Aspirierte Fremdkörper können je nach Art und v. a. Lokalisation in den oberen Atemwegen relativ schnell zu einer dramatischen Notfallsituation führen. Die Versorgungssituation bietet in Deutschland allerdings weiterhin ein recht buntes Bild. Während die Gastroenterologie mit ihren flexiblen Endoskopiemöglichkeiten eine nahezu flächendeckende Versorgungsstruktur aufgebaut hat und je nach lokaler Versorgungssituation auch primär kontaktiert wird, ist die Versorgung mit interventionell tätigen Pneumonologen in der Breite nicht gegeben, sodass hier regelmäßig die HNO-Kliniken involviert werden. Da bei den aspirierten Fremdkörpern sehr viel häufiger Kinder betroffen sind, finden sich auch pädiatrische Kliniken mit einer bronchoskopisch interventionellen Expertise. Nach wie vor verfügen aber die meisten der fast $150 \mathrm{HNO}$-Hauptabteilungen, der 36 HNO-Universitätskliniken und einige HNO-Belegabteilungen über das endoskopische Know-how mit flexiblen und starren Endoskopietechniken zur Entfernung ingestierter und aspirierter Fremdkörper. Hinzu kommt, dass in den HNOKliniken eine sichere Expertise in der Kinderanästhesie vorgehalten wird.

Im vorliegenden Themenheft werden aktuelle Aspekte der Indikationsstellung und endoskopischen Entfernung aspirierter und ingestierter Fremdkörper von $\mathrm{Au}$ toren verschiedener Fachdisziplinen diskutiert. In einem Literaturupdate werden neue technische Entwicklungen der interventionellen Endoskopie ebenso wie die Frage diskutiert, wann ein ingestierter Fremdkörper beobachtet werden kann.

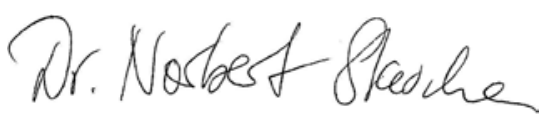

N. Stasche

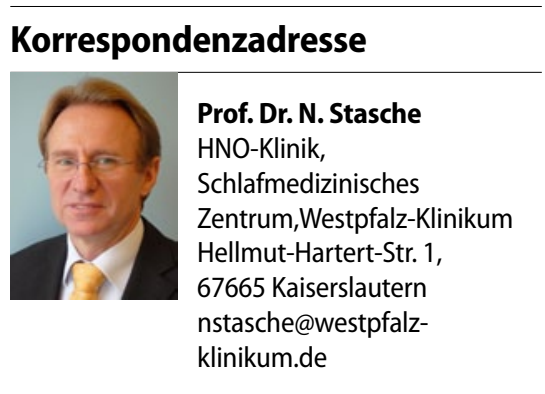

